Available online at GSC Online Press Directory

GSC Biological and Pharmaceutical Sciences

e-ISSN: 2581-3250, CODEN (USA): GBPSC2

Journal homepage: https://www.gsconlinepress.com/journals/gscbps

(REVIEW ARTICLE)

\title{
Bilayer tablet technology: A novel approach
}

\author{
Sadhu Venkateswara Rao ${ }^{1, *}$, Bopparaju Priyanka ${ }^{1}$ and Kantamneni Padmalatha ${ }^{2}$ \\ ${ }^{1}$ Department of Pharmaceutics, Vijaya Institute of Pharmaceutical Sciences for Women, Enikepadu, Vijayawada-521108, \\ India. \\ ${ }^{2}$ Department of Pharmacology, Vijaya Institute of Pharmaceutical Sciences for Women, Enikepadu, Vijayawada-521108, \\ India.
}

Publication history: Received on 28 February 2019; revised on 26 April 2019; accepted on 04 May 2019

Article DOI: https://doi.org/10.30574/gscbps.2019.7.2.0033

\begin{abstract}
Bilayer tablet is new era for the successful development of controlled release formulation along with various features to provide a way of successful drug delivery system. Controlled release dosage forms have been extensively used to improve therapy with several important drugs. Use of bilayer tablet is a very different aspect for anti-inflammatory and analgesic. Bilayer tablet is suitable for sequential release of two drugs in combination, separate two incompatible substances and also for sustained release tablet in which one Layer is immediate release as initial dose and second layer is maintenance dose. Bilayer tablet is improved beneficial technology to overcome the shortcoming of the single layered tablet. This article provides an overview of the bilayer tablet technology, highlighting the main benefits of this type of oral dosage forms while providing a description of current challenges and advances toward improving manufacturing practices and product quality.
\end{abstract}

Keywords: Bilayer tablet; Sustained release; Immediate release; Incompatible

\section{Introduction}

Over the past 30 years greater attention has been focused on development of sustained or controlled release drug delivery systems. The development of combination of two or more active pharmaceutical ingredients (API) in a single dosage form has increased in the pharmaceutical industry, promoting patient convenience and compliance [1]. Bilayer tablet is new era for the successful development of controlled release formulation along with various features to provide a way of successful drug delivery system. Bilayer tablet is suitable for sequential release of two drugs in combination, separate two incompatible substances and also for sustained release tablet in which one layer is immediate release as initial dose and second layer is maintenance dose [2].

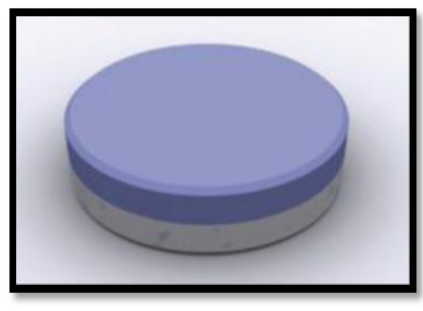

\footnotetext{
${ }^{*}$ Corresponding author

E-mail address: venkateshsadhu@gmail.com
} 
Figure 1 Bilayer tablet [14]

\title{
Need of bilayer tablets
}

1. Controlling the delivery rate of single or two different active pharmaceutical ingredients.

2. To modify the surface area available for API by swellable/erodible barriers for modified release.

3. To separate incompatible Active pharmaceutical ingredient (APIs) from each other.

4. To control the release of API from one layer by utilizing the functional property of the other layer.

5. For the administration of fixed dose combinations of different APIs.

\author{
Advantages \\ 1. Cost is subordinate compared to all other oral dosage form. \\ 2. Maximum chemical and microbial stability over all oral dosage form \\ 3. Offensive odor and bitter taste can be masked by coating technique. \\ 4. Flexible Concept. \\ 5. They offer greatest dose accuracy and least content variability. \\ 6. Easy to swallowing with least tendency for hang up. \\ 7. Suitable for large scale production
}

\section{Disadvantages}

1. Some drugs resist compression into dense compacts due to low density character.

2. Bitter tasting drugs or drugs that are sensitive to oxygen may require coating.

3. Difficult to swallow in case of children and unconscious patients.

4. Drugs with poor wetting, slow dissolution properties may be difficult to formulate.

\section{Ideal characteristics of bilayer tablets}

1. It should be free from defects like chips, cracks, discoloration and contamination.

2. It should have sufficient strength during its production, packaging, shipping and dispensing.

3. It should have the chemical and physical stability overtime.

4. It releases the agents in a predictable and reproducible manner.

5. It must have a chemical stability and shelf-life.

\section{Challenges in bilayer tablet manufacturing}

Conceptually, bilayer tablets can be seen as two single-layer tablets compressed into one. In Practice, there are some manufacturing challenges [3].

Delamination: Tablet falls apart when the two halves of the tablet do not bond completely.

Cross-Contamination: When the granulation of the first layer intermingles with the granulation of the second layer results cross contamination occurs. Proper dust collection goes a long way toward preventing cross contamination.

Production yields: To prevent cross contamination, dust collection is required which leads to losses. Thus, bilayer tablets have lower yields than single layer tablets.

Cost: Bilayer tableting is more expensive than single layer tableting for several reasons. First, the tablet press costs more. Second, the press generally runs more slowly in bilayer mode. Third, development of two compatible granulations is must, which means more time spent on formulation development, analysis and validation [4].

\section{Types of bilayer tablet press}

\subsection{Single sided tablet press}

The simplest design is a single sided press with both chambers of the doublet feeder separated from each other. Each chamber is gravity or forced fed with different power, producing the two individual layers of tablets. When die passes under the feeder, it is first loaded with the first layer powder followed by the second layer powder. Then the entire tablet is compressed in one or two steps [5]. 


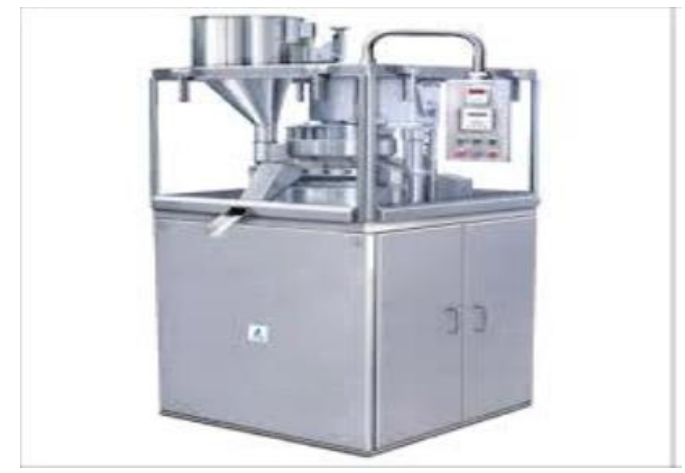

Figure 2 Single sided tablet press [15]

\section{Limitations of the single sided press}

1. No weight monitoring / control of the individual layers.

2. No distinct visual separation between the two layers.

3. Capping and hardness problems.

\subsection{Double sided tablet press}

In most double sided tablet presses with automated production control use compression force to monitor and control tablet weight. The compression force exerted on each individual tablet or layer is measured by the control system at main compression of the layer [6]. This compression force is the signal used by the control system to reject out of tolerance and correct the die fill depth when required.

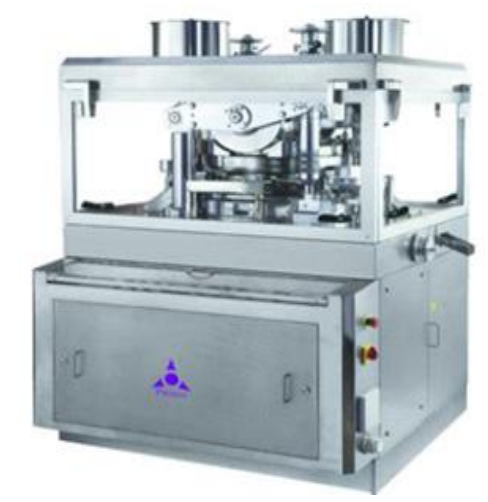

Figure 3 Double sided tablet press [16]

\subsection{Bilayer tablet press with displacement monitoring}

The displacement tablet weight control principle is fundamentally different from the principle based upon compression force. When measuring displacement, the control system sensitivity does not depend on the tablet weight but depends on the applied precompression force [7].

\section{Advantages}

1. Weight monitoring / control weight of the individual layers.

2. Avoid capping and separation of the two individual layers.

3. Independence from the machine stiffness.

4. Provide sufficient hardness at maximum turret speed.

5. Maximum prevention of cross-contamination between the two layers.

6. Clear visual separation between the two layers and maximized yield. 


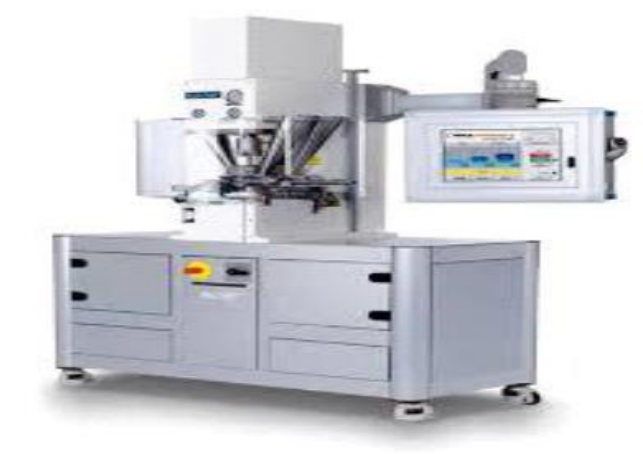

Figure 4 Bilayer tablet press with displacement monitoring [17]

\section{Preparation of bilayer tablets}

Bilayer tablets are prepared with one layer of drug for immediate release and second layer designed to release drug either as a second dose or in an extended release form. To produce adequate tablet formulation, certain requirements such as sufficient mechanical strength and desired drug release profile must be met. At times, this may be difficult task for formulator to achieve these conditions especially in bilayer tablet formulation, where double compression technique is involved, because of poor flow and compatibility characteristic of the drug which will result in capping and/or lamination [8].

Compaction: The process by which the porosity of a given powder is decreased as a result of its grains being squeezed together by the weight of mechanical means. The compaction of a material involves both the compressibility and consolidation.

Compression: It is defined as reduction in bulk volume by eliminating voids and bringing particles into closer contacts.

Consolidation: It is the property of the material in which there is increased mechanical strength due to inter particulate interaction (bonding). The compression force on layer 1 was found to be major factor influencing tablet delamination.

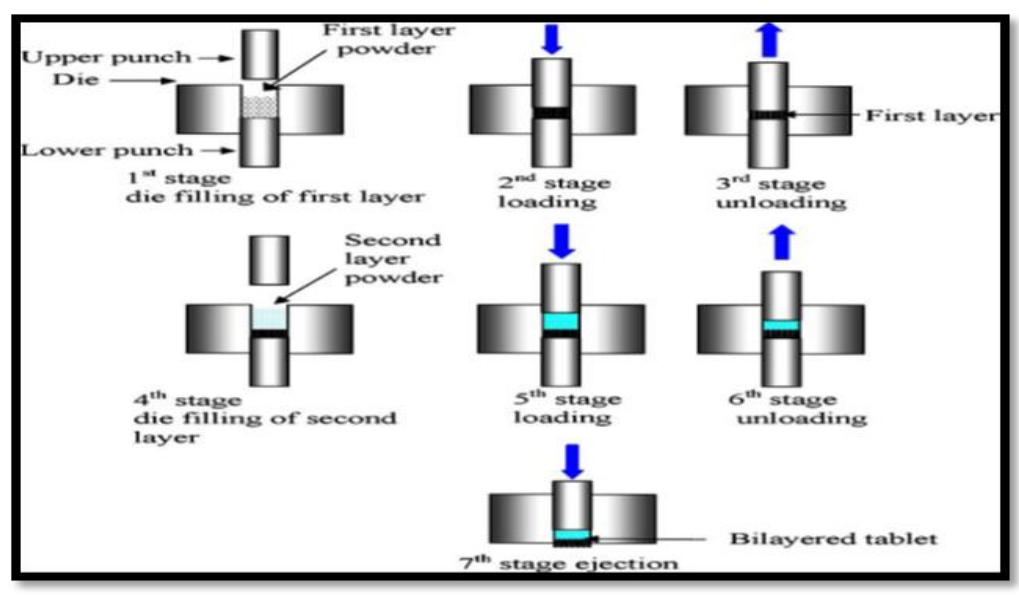

Figure 5 Production of bilayer tablets [18]

\section{Various approaches used in the bilayer tablet}

\subsection{Floating Drug Delivery System}

From the formulation and technological point of view, the floating drug delivery systems are considerably easy and logical approach in the development of Gastro retentive dosage forms (GRDFs). 
Approaches to design Floating Drug Delivery System

The following approaches have been used for the design of floating dosage forms of single and multiple-unit systems.

\subsubsection{Intra gastric bilayered floating tablets}

These are also compressed tablet as shown in figure and contain two layers i.e. Immediate and sustained release.

\subsubsection{Multiple unit type floating pills}

These systems consist of sustained release pills as 'seeds' surrounded by double layers. The inner layer consists of effervescent agents while the outer layer is of swellable membrane layer. When the system is immersed in dissolution medium at body temperature, it sinks at once and then forms swollen pills like balloons, which float as they have lower density [9].

\subsection{Polymeric Bio adhesive System}

These are designed to imbibe fluid following administration such that the outer layer becomes a viscous, tacky material that adheres to the gastric mucosa/mucus layer. This should encourage gastric retention until the adhesive forces are weakened. These are prepared as one layer with immediate dosing and other layer with bio adhesive property [10].

Disadvantages: The success is seen in animal models with such system has not been translated to human subjects due to differences in mucous amounts, consistency between animals and humans.

\subsection{Swelling System}

These are designed to be sufficiently small on administration so as not to make ingestion of the dosage form difficult. On ingestion they rapidly swell or disintegrate and passage through the pylorus until after drug release has progressed to a required degree. The simple bilayer tabletmay contain an immediate release layer with the other layer as extended release [11].

\section{Evaluation of bilayer tablets}

General appearance: The general appearance of a tablet, its visual identity and overall "elegance" is essential for consumer acceptance. Includes in are tablet's size, shape, colour, presence or absence of an odour, taste and surface texture.

Size and shape: The size and shape of the tablet can be dimensionally described, monitored and controlled.

Tablet thickness: Ten tablets were taken and their thickness was recorded using micrometer.

Weight variation: Standard procedures are followed as described in the official books.

Friability: The friability test is closely related to tablet hardness and is designed to evaluate the ability of the tablet to withstand abrasion in packaging, handling and shipping. It is usually measured by the use of the Roche friabilator [12].

$\%$ Friability = 1- (loss in weight / Initial weight) X 100

Hardness (Crushing strength): The resistance of tablets to capping, abrasion or breakage under conditions of storage, transportation and handling before usage depends on its hardness. The small and portable hardness tester Monsanto or Stokes hardness tester are used to measure the hardness of the tablet.

Dissolution: Standard procedures are followed as described in the official books.

Stability study: The bilayer tablets are packed in suitable packaging and stored under different temperature conditions for a period as prescribed by ICH guidelines for accelerated studies [13]. 


\section{Conclusion}

Bilayer tablet is new novel of tablet for the successful development of controlled release formulation along with many features to provide a way of successful drug delivery system. Bilayer tablet is improved beneficial technology to overcome the shortcoming of the single layered tablet. Bi-layer tablet is suitable for sequential release of two drugs in combination, separate two incompatible substances and also for sustained release tablet in which one Layer is immediate release as initial dose and second layer is maintenance dose. So use of bi-layer tablets is a very different aspect for anti-hypertensive, diabetic, anti-inflammatory and analgesic drugs where combination therapy is often used.

\section{Compliance with ethical standards}

\section{Acknowledgments}

The presenting authors are thankful to principal, Vijaya institute of pharmaceutical sciences for women, Vijayawada for their valuable support in carrying out this work.

\section{Disclosure of conflict of interest}

All authors declare that they have no conflict of interest.

\section{References}

[1] Martindale. (1996). The Extra Pharmacopoeia. 31 $1^{\text {st }}$ ed. The Pharmaceutical Press, London, 936-937.

[2] Naisarg D, Pujararonak K, Gokani and Jalpa S. (2011). Bilayer tablet - An emerging trend. International Journal of Pharmaceutical Research and Development, 4(04), 102 - 111

[3] Varaiya C. (2005). Bi-layer neutraceutical tablets: Rewards and challenges. Journal of Pharmacy and Pharmacology, 40, 282 - 283

[4] Panchal HA and Tiwari AK. (2012). A Novel approach of bilayer tablet technology-A review, International Research Journal of Pharmacy, 3(5), 152 - 156

[5] Patel M, Sockan GN and Kavitha MT (2010). Challenges in the formulation of bilayered tablets: A review. International Journal of Pharma Research and Development, 0974 - 9446.

[6] Singh PK and Kumar S. (2011). Bilayer and floating bioadhesive tablets: Innovative approach to gastro retention. Journal of Drug Delivery \& Therapeutics, 1(1), 32-35.

[7] Hamachek TR. (2002). Innovative oral drug delivery technologies, Penwest pharmaceuticals. The Pharmaceutical Press, London. 936-937

[8] Li SP, Karth MG, Feld KM, Pendharkar CM and Willams RO. (1995). Evaluation of bilayer tablet machines: A Case study. Drug Developmentand Industrial Pharmacy, 21(5), 571-590.

[9] Shirwalkar AA, Kumar SM and Jacob S. (2006). Recent developments in floating drug delivery systems for gastric retention of drugs, an overview. Indian drugs, 43(9), 697-704.

[10] Verma RK and Garg S. (2001). Drug delivery technologies and future directions. Pharmaceutical Technology, 25(2), 1-14.

[11] Breech AJ, Lucisano L and Franz RM. (1998). Investigation into substrate cracking of a film coated bilayered tablet. Journal of Pharmacy and Pharmacology, 40, 282 - 283

[12] Nirmal J, Saisivam S, Peddanna C, Muralidharan S and Nagarajan M. (2008). Bilayer tablets of atorvastatin calcium and nicotinic acid: formulation and evaluation. Chemical and Pharmaceutical Bulletin, 56, 1455-1458.

[13] Shiyani B. (2008). Formulation and evaluation of bilayer tablet of Metoclopramide hydrochloride and Ibuprofen. AAPS Pharm Sci Tech, 9(3), 818-27.

[14] Sharma SK, Mohan S, Jaimini M and Tiwari R. (2014). Polytherapeutic approach using bilayer matrix Technology. Asian Journal of Pharmaceutics, 30, 225 - 254.

[15] Single sided tablet press image. https://www.google.com/search?q=Single+sided+tablet+press\&source

[16] Double sided tablet press. https://www.google.com/search?biw=1152\&bih=735\&tbm=Double+sided 
[17] Bilayer tablet press with displacement monitoring https://www.google.com/search?biw=1152\&bih

[18] Gopinath C, Bindu VH and Nischala M. (2013). An overview on bilayered tablet technology. Journal of global trends in pharmaceutical sciences, 4(2), 1077-1085.

\section{How to cite this article}

Sadhu VR, Bopparaju P and Kantamneni P. (2019). Bilayer tablet technology: A novel approach. GSC Biological and Pharmaceutical Sciences, 7(2), 22-28. 